\title{
WEIGHTED NORM INEQUALITIES FOR FRACTIONAL INTEGRALS
}

\author{
G. V. WELLAND ${ }^{1}$
}

ABSTRACT. A simpler proof of an inequality of Muckenhoupt and Wheeden is given. Let $T_{a} f(x)=\int f(y)|x-y|^{a-d} d y$ be given for functions defined in $\mathrm{R}^{d}$. Let $v$ be a weight function which satisfies

$$
\left(|Q|^{-1} \int_{Q}[v(x)]^{q} d x\right)^{1 / q}\left(|Q|^{-1} \int_{Q}[v(x)]^{-p^{\prime}} d x\right)^{1 / p^{\prime}} \leq K
$$

for each cube, $Q$, with sides parallel to a standard system of axes and $|Q|$ is the measure of such a cube. Suppose $1 / q=1 / p-a / d$ and $0<a<d, 1<$ $p<d / a$. Then there exists a constant such that $\left\|\left(T_{a} f\right) v\right\|_{q} \leq C\|f v\|_{p}$. Certain results for $p=1$ and $q=\infty$ are also given.

1. In this note we present a proof of a result of Muckenhoupt and Wheeden. The technique used is suggested by Hedberg [2]. We also give some results which parallel those in [2] and [3] for the cases $p=1$ and $q=\infty$.

A nonnegative function $v$, defined on $\mathbf{R}^{d}$, is said to belong to $A_{p, q}(1$ $<p, q<\infty)$ if

$$
\left(\frac{1}{|Q|} \int_{Q} v^{q}(x) d x\right)^{1 / q}\left(\frac{1}{|Q|} \int_{Q} v(x)^{-p^{\prime}} d x\right)^{1 / p^{\prime}} \leq K<\infty .
$$

Here $p^{\prime}=p /(p-1)$ and $Q$ denotes a $d$-dimensional cube in $\mathbf{R}^{d}$ with sides parallel to a standard system of axes and centered at $x$. For $p=1$, this condition becomes

$$
\left(\frac{1}{|Q|} \int_{Q} v^{q}(x) d x\right)^{1 / q} \leq K \inf _{y \in Q}[v(y)]
$$

For $q=\infty$, the condition (1.1) becomes

$$
\left(\frac{1}{|Q|} \int_{Q} v(x)^{-p^{\prime}} d x\right)^{1 / p^{\prime}} \leq K \inf _{y \in Q}\left[\frac{1}{v(y)}\right]
$$

All suprema and infima are essential.

The fractional integral operator is

$$
T_{a} f\left(x_{0}\right)=\int_{\mathbf{R}^{d}} f(y)|x-y|^{a-d} d y .
$$

Received by the editors May 28, 1974.

AMS (MOS) subject classifications (1970). Primary 26A33, 26 A86.

'supported by NSF Grant GP-34360. 
We will also be concerned with certain maximal operators $(0 \leq a<d)$;

$$
\left(M_{a} f\right)(x)=\sup _{Q \subset \mathrm{R}^{d}} \frac{1}{|Q|^{1-\alpha / d}} \int_{Q}|f(y)| d y,
$$

where the supremum is taken over cubes centered at $x$.

Lemma $1[3,(2.4)]$. Assume that $0<a<d, 1<p<d / \alpha, 1 / q=1 / p-$ $a / d$ and $v \in A_{p, q^{*}}$ Then there exists a constant $C$ which is independent of $f$ such that

$$
\left\{\int_{\mathbf{R}^{d}}\left|\left(M_{a} f\right)(x) v(x)\right|^{q} d x\right\}^{1 / q} \leq C\left(\int_{\mathbf{R}^{d}}|f(x) v(x)|^{p} d x\right)^{1 / p} .
$$

For $\alpha=0$ and $v \equiv 1$ this becomes the usual Hardy-Littlewood maximal theorem.

The proof of Lemma 1 is based on the Marcinkiewicz interpolation theorem [4, Volume II, p. 112] and weak type estimates for the operator $T g$ on the measure spaces $\left(\mathbf{R}^{d}, d \mu\right)$ where $d \mu=\nu^{q}(x) d x$ and

$$
(T g)(x)=\left(M_{a}\left(g\left(v^{q}\right)^{\alpha / d}\right)\right)(x) .
$$

In particular, if $v \in A_{p, q}$, then

$$
\int_{T \boldsymbol{g}>a} v^{q}(x) d x \leq C\left\{\frac{1}{a} \int_{\mathbf{R}^{d}}|g(x)|^{p} v^{q}(x) d x\right\}^{q / p} .
$$

The key point to the use of the Marcinkiewicz interpolation theorem is the fact that $v \in A_{p, q}$ implies $v \in A_{p_{i}, q_{i}}, i=1,2$, where $1 / q_{i}=1 / p_{i}-\alpha / d$ and $1<p_{1}<p<p_{2}$. The possible values of $p_{1}$ and $p_{2}$ depend on the function $v$. This fact follows from the "reverse" Hölder condition [1, Theorem IV]. In case $v \in A_{1}, q_{1}$ where $1<q_{1}<\infty$, the reverse Hölder condition again can be obtained and one can show there is a $p_{0}>1$ such that $v \epsilon$ $A_{p_{0}, q_{0}}$ where we use $1 / q_{0}=1 / p_{0}-\alpha / d$. In this case, the interpolation theorem leads to (1.4) with the constant $C=C_{t}=O(1 /(1-t))$ as $t \rightarrow 1$ where $1 / p=t+(1-t) / p_{0}$ and $1 / q=t / q_{1}+(1-t) / q_{0}$. Using $g(x)=$ $f(x)(w(x))^{-\alpha / d}$ and $w(x)=v^{q}(x),(1.4)$ can be written as

$$
\left[\int|(T g)(x)|^{q} w(x) d x\right]^{1 / q} \leq C_{t}\left[\int|g(x)|^{p} w(x) d x\right]^{1 / p} .
$$

When $p=1$ we have the following typical result.

Proposition 1. Assume $p=1,1 / q=1-\alpha / d, v \in A_{1, q}, f$ has support in a ball $B$, and $\int_{B}|f(x)| \log ^{+}\left|f(x) v^{1-q}(x)\right| v(x) d x<\infty$. Then 


$$
\begin{aligned}
& \left\{\int_{B}\left|\left(M_{\alpha} f\right)(x) v(x)\right|^{q} d x\right\}^{1 / q} \\
& \leq C\left[w(B)+\int_{B}|f(x)| \log ^{+} \mid f(x) v^{\left.1-q_{1}(x) \mid v(x) d x\right]}\right.
\end{aligned}
$$

where $w(B)=\int_{B} w(x) d x$.

Proof. The proof follows the lines of that in [4]. Let $g(x)=$ $f(x) v(x)^{-q a / d}, E_{k}=\left\{x \in B\left|2^{k} \leq\right| g(x) \mid<2^{k+1}\right\}, k=0,1,2, \cdots, g_{k}(x)=$ $\chi_{E_{k}}(x) g(x)$ where $\chi_{E_{k}}$ is the characteristic function of $E_{k}$, and let $1 / p_{k}=$ $t_{k}+\left(1-t_{k}\right) / p_{0}, 1 / q_{k}=1 / p_{k}-\alpha / d$ where $t_{k}=(k+1) /(k+2)$. Then by using Minkowski's inequality, Hölder's inequality and then (1.5') one obtains

$$
\begin{aligned}
\left\{\int_{B}|T g(x)|^{q} w(x) d x\right. & \}^{1 / q} \leq \sum_{k=0}^{\infty}\left\{\int_{B}\left|\left(T g_{k}\right)(x)\right|^{q} w(x) d x\right\}^{1 / q} \\
& =\sum_{k=0}^{\infty} w(B)^{1 / q}\left\{\frac{1}{w(B)} \int_{B}\left|T g_{k}(x)\right|^{q} k w(x) d x\right\}^{1 / q_{k}} \\
& \leq \sum_{k=0}^{\infty} w(B)^{1-1 / p_{k}} C_{t_{k}}\left\{\int_{B}\left|g_{k}(x)\right|^{p_{k}} w(x) d x\right\}^{1 / p_{k}} \\
& \leq C w(B) \sum_{k=0}^{\infty}(k+2) w(B)^{-1 / p_{k}} 2^{k+1}\left(\int_{E_{k}} w(x) d x\right)^{1 / p_{k}} \\
& =C w(B) \sum_{k=0}^{\infty}(k+2)\left[\frac{w\left(E_{k}\right)}{w(B)}\right]^{1 / p_{k}} 2^{k+1} .
\end{aligned}
$$

Let $K$ be the set of nonnegative integers for which $\left[w\left(E_{k}\right) / w(B)\right]^{1 / p_{k}}$ $\leq 3^{-(k+1)}$. Let $K^{\prime}$ be the complement of $K$ in the nonnegative integers. For $k \in K^{\prime}$, noting $w\left(E_{k}\right) / w(B) \leq 1$, it follows that there is a constant $C$ such that $\left[w\left(E_{k}\right) / w(B)\right]^{1 / p_{k}-1} \leq C$. Using this and the above inequality we have

$$
\begin{aligned}
\left\{\int_{B}|T g(x)|^{q} w(x) d x\right\}^{1 / q} & \leq C w(B) \sum_{k \in K}(k+2)\left(\frac{2}{3}\right)^{k+1}+C \sum(k+2) 2^{k+1} w\left(E_{k}\right) \\
& \leq C\left[w(B)+\int_{B}|g(x)| \log ^{+}|g(x)| w(x) d x\right] .
\end{aligned}
$$

By substituting the definition of $T$ and $g$ in the above inequalities (1.6) results.

2. In this section we prove

Theorem 1. Assume $0<\alpha<d, 1<p<n / a, 1 / q=1 / p-\alpha / d$ and $v \epsilon$ 
$A_{p, q^{\cdot}}$ Then there exists a constant $C$ independent of $f$ such that

$$
\left[\int_{\mathbf{R}^{d}}\left|T_{a} f(x) v(x)\right|^{q} d x\right]^{1 / q} \leq C\left[\int_{\mathbf{R}^{d}}|f(x) v(x)|^{p} d x\right]^{1 / p} \text {. }
$$

In case $p=1$, suppose $f$ has support in the ball $B$. Then

$$
\left[\int_{B}\left|T_{a} f(x) v(x)\right|^{q} d x\right]^{1 / q} \leq C\left[w(B)+\int_{B}|f(x)| \log ^{+}\left|f(x) v^{1-q}(x)\right| v(x) d x\right]
$$

where $w(B)=\int_{B} v^{q}(x) d x$.

We remark here that in [3] it is proved that $v \in A_{p, q}$ is both necessary and sufficient for (2.1). The proof here is somewhat simpler. Finally they give a weak type estimate instead of (2.2) for which $v \in A_{1, q}$ is necessary and sufficient.

Proof. Let $\delta>0$ be given. Then

$$
\begin{aligned}
T_{a} f(x) & =\int_{|x-y|<\delta} f(y)|x-y|^{a-d} d y+\int_{|x-y| \geq \delta} f(y)|x-y|^{a-d} d y \\
& =I_{1}(x)+I_{2}(x) .
\end{aligned}
$$

Let $R_{i}=\left\{y\left|2^{-i-1} \delta \leq\right| x-y \mid<2^{-i} \delta\right\}$ where $i$ is any integer. We have

$$
\begin{aligned}
\left|I_{1}(x)\right| & \leq \sum_{i=0}^{\infty} \int_{R_{i}}|f(y)| \cdot|x-y|^{a-d} d y \leq \sum_{i=0}^{\infty}\left(2^{-i-1} \delta\right)^{a-d} \int_{R_{i}}|f(y)| d y \\
& \leq \sum_{i=0}^{\infty} 2^{d-a}\left(2^{-i}\right)^{\epsilon} \delta^{\epsilon} \frac{1}{\left(2^{-i} \delta\right)^{d-a+\epsilon}} \int_{B_{i}}|f(y)| d y
\end{aligned}
$$

where $B_{i}=\left\{y|| x-y \mid \leq 2^{-i} \delta\right\}$. Hence $I_{1}(x) \leq A_{\epsilon} \delta^{\epsilon}\left(M_{a_{-} \epsilon} f\right)(x)$. Similarly

$$
I_{2}(x) \leq \sum_{i=1}^{\infty}\left(2^{i-1} \delta\right)^{a-d} \int_{B_{-i}}|f(y)| d y \leq A_{\epsilon}^{\prime} \delta^{-\epsilon}\left(M_{a+\epsilon} f\right)(x) .
$$

By choosing $\delta^{\epsilon}=\left[\left(M_{a+\epsilon} f\right)(x) /\left(M_{a-\epsilon} f\right)(x)\right]^{1 / 2}$ one finds

$$
\left|T_{a} f(x)\right| \leq A\left[\left(M_{a+\epsilon} f\right)(x)\left(M_{a-\epsilon} f\right)(x)\right]^{1 / 2} \text {. }
$$

Let $1 / q_{\epsilon}=1 / p-(\alpha+\epsilon) / d$ and $1 / \bar{q}_{\epsilon}=1 / p-(\alpha-\epsilon) / d$. Noting that $v \epsilon$ $A_{p, q}$ implies $v \in A_{p, \bar{q}_{\epsilon}}$ and $v \in A_{p, q_{\epsilon}}$ provided we choose $\epsilon$ small enough, we can now apply (1.4) for the operators $M_{a+\epsilon}$ and $M_{a-\epsilon}$. Let

$$
F=\left[\left(M_{a+\epsilon} f\right)(x) v(x)\right]^{q / 2} \text { and } G=\left[\left(M_{a-\epsilon} f\right)(x) v(x)\right]^{q / 2} \text {. }
$$

Let $p_{1}=2 q_{\epsilon} / q$ and $p_{2}=2 \bar{q}_{\epsilon} / q$ so that $1 / p_{1}+1 / p_{2}=1$. Applying Hölder's inequality to (2.3) after integrating and using the above notation, we have 


$$
\int\left|T_{a} f(x) v(x)\right|^{q} d x \leq\|F\|_{p_{1}}\|G\|_{p_{2}} .
$$

By an application of (1.4)

$$
\|F\|_{p_{1}}=\left\{\int\left|\left(M_{a+\epsilon} f\right)(x) v(x)\right|^{q_{\epsilon}} d x\right\}^{1 / p_{1}} \leq C\|f v\|_{p}^{q_{\epsilon} / p_{1}} .
$$

Similarly $\|G\|_{p_{2}} \leq C\|f v\|_{p}^{\bar{q}_{t} / p_{2}}$. Combining these inequalities leads to (2.1).

The proof of (2.2) follows in the same manner with the use of inequality (1.6).

We give one more result, this time, for the case $q=\infty$.

Theorem 2. Let $f$ have support in a ball $B$ of radius $R$. Let $Q$ be the smallest cube containing $B$. Assume $d=p a, 1<p<\infty$, and that $v \in A_{p, \infty}$. Then for $\epsilon>0$, and $p<q<\infty$, and $m=$ inf $_{x \in Q} v(x)$, there exists a constant $C=C(\epsilon, q)$ such that

$$
\int_{B} \exp \left[\frac{d}{w_{d-1}}\left|\frac{m\left|T_{a} f(x)\right|}{\|f v\|_{p}}-\epsilon\right|^{p^{\prime}}\right] v^{q}(x) d x \leq C R^{d_{m}}{ }^{q} .
$$

Here $w_{d-1}$ is the area of the surface of the unit ball in $\mathbf{R}^{d}$.

Proof. The proof follows [2]. For $q$ given define $\sigma$ by $1 / q=1 / p-$ $(a-\sigma) / d$. Let $\delta>0$ and assume $f$ is such that $\|f v\|_{p} \leq m$. As before $T_{a} f(x)=I_{1}(x)+I_{2}(x)$ and $\left|I_{1}(x)\right| \leq A_{\sigma} \delta^{\sigma} M_{a_{-} \sigma} f(x)$ and for $x \in B$

$$
\begin{aligned}
\left|I_{2}(x)\right| & \leq\left|\int_{|x-y| \geq \delta} v(y) f(y)\right| x-\left.y\right|^{a-d} \frac{d y}{v(y)} \mid \\
& \leq\|f v\|_{p} \cdot\left[\sup _{y \in Q}[v(y)]^{-1}\right]\left[w_{d-1} \log \frac{R}{\delta}\right]^{1-1 / p} \leq\left[w_{d-1} \log \frac{R}{\delta}\right]^{1 / p^{\prime}} .
\end{aligned}
$$

Choose $\delta^{\sigma}=\min \left\{\epsilon\left[A_{\sigma} M_{\alpha_{-} \sigma} f(x)\right]^{-1}, R^{\sigma}\right\}$ so that

$$
\left|T_{a} f(x)\right| \leq \epsilon+\left\{w_{d-1} \log ^{+} R \epsilon^{-1 / \sigma}\left[A_{\sigma} M_{a-\sigma} f(x)\right]^{1 / \sigma}\right\}^{1 / p^{\prime}}
$$

and, hence,

$$
|| T_{a} f(x)|-\epsilon|^{p^{\prime}} \leq\left(w_{d-1} / d\right)\left\{\log ^{+} R^{d_{\epsilon}-q}\left[A_{\sigma} M_{a-\sigma} f(x)\right]^{q}\right\}_{.}
$$

From this it follows that

$$
\begin{aligned}
& \int_{B} \exp \left[\left(d / w_{d-1}\right) \| T_{a} f(x)|-\epsilon|^{p^{\prime}}\right] v^{q}(x) d x \\
& \quad \leq R^{d} \epsilon^{-q} A_{\sigma}^{q} \int_{q}\left|M_{a-\sigma} f(x)\right|^{q} v^{q}(x) d x \leq R^{d} C\|f v\|_{p}^{q} .
\end{aligned}
$$

The last inequality follows from (1.4). 
To obtain (2.4) in general, we use (2.5) with the function $g(x)=$ $f(x) m\|f v\|_{p}^{-1}$.

Remarks. By checking the constants, one finds that $C \leq(A q / \epsilon d)^{q}$ where $A$ is a constant times the $K$ which appears in (1.3). From this and an application of Stirling's formula one obtains

(2.4') $\quad \int_{B} \exp \left[\frac{d}{w_{d-1}}\left|\frac{m\left|T_{a} f(x)\right|}{\|f v\|_{p}}-\epsilon\right|^{p^{\prime}}+\frac{v(x) \epsilon d}{A m} \cdot \frac{1}{e+\epsilon^{\prime}}\right] d x \leq C\left(\epsilon^{\prime}\right) R^{d}$

where $C\left(\epsilon^{\prime}\right)<\infty$ for each $\epsilon^{\prime}>0$.

Suppose $f$ is a function such that for any cube $Q_{i}\left(\chi_{Q_{i}}\right.$ is the characteristic function'of $\left.Q_{i}\right)\left\|v f \chi_{Q_{i}}\right\|_{p} \leq\left|Q_{i}\right| \sup _{y} \epsilon_{i}|v(y)|$. Then simple estimates using (1.3) result in

$$
\left|I_{2}(x)\right| \leq K\left[w_{d-1} \log (R / \delta)\right]
$$

without an introduction of the number $m$. Use of this inequality leads to

$$
\int_{B} \exp \left[\left(d / K w_{d-1}\right) \| T_{a} f(x)|-\epsilon|^{p^{\prime}}\right] \nu^{q}(x) d x \leq C R^{d}\|f v\|_{p}^{q} .
$$

\section{REFERENCES}

1. R. R. Coifman and C. Fefferman, Weighted norm inequalities for maximal functions and singular integrals, Studia Math. 51 (1974), 241-250.

2. L. I. Hedberg, On certain convolution inequalities, Proc. Amer. Math. Soc. 36 (1972), 505-510. MR. 47 \#794.

3. B. Muckenhoupt and R. L. Wheeden, Weighted norm inequalities for fractional integrals, Trans. Amer. Math. Soc. 192 (1974), 261-274.

4. A. Zygmund, Trigonometric series. Vols. I, II, 2nd ed., Cambridge Univ. Press, New York, 1959. MR 21 \#6498.

5. —_, A note on the differentiability of integrals, Colloq. Math. 16 (1967), 199-204. MR $35 \# 1732$.

DEPARTMENT OF MATHEMATICS, UNIVERSITY OF MISSOURI-ST. LOUIS, ST. LOUIS, MISSOURI 63130 